\title{
The Role of Criminal Law as an Anti-corruption Tool in the Public Sector
}

\author{
Kesiana Çoçka
}

Phd. Candidate, Law Faculty of Tirana University, Department of Civil Law Str. "Milto Tutulani", Tiranë, Albania; kesiana_cocka@yahoo.com

\section{Prof. Asoc. Dr. Ilir Rusi}

Law Faculty of Tirana University, Department of Civil Law Str. "Milto Tutulani", Tiranë, Albania; Email:ilirusi@yahoo.com

\section{Doi:10.5901/ajis.2016.v5n1p99}

\begin{abstract}
The Albanian society is making efforts for the consolidation of democratic institutions and the fulfillment of standards as a condition for European integration. In the context of these efforts, the fight against corruption remains a major challenge. The need to address the fight against it is one of the main issues of public interest. Corruption in the field of public administration is one of the most disturbing phenomena of the Albanian society. European Commission Progress Report 2011 for Albania considers public administration reform as one of the key priorities. To this end, the Albanian government has undertaken concrete steps to combat and prevent corruption in the public sector in particular. Concrete steps in the context of the fight against corruption,consists on a very important place which is given to legal reform, especially the amendment of criminal legislation as an important war of the fight against corruption. Although the legal framework has improved significantly, there has been no proper success in changing the reality in relation to the prevention and suppression of corruption. Studies and surveys indicate an improvement of the situation, but the corruption practices in the public sector continue to be embodied as the economic dimension, as well as in social. The purpose of this paper is to present some issues regarding anticorruption legal reform in the field of public administration based on an analysis of international law, provisions of the Criminal Code and special laws aimed at preventing and combating corruption in the public sector. More specifically, the paper will focus on: Analysis of the Criminal Code amendments that occurred as a result of demand for harmonization of legislation with international conventions in this field, and the importance in combating corruption in the public sector. - Analysis of legislation and other measures in the field of public administration that enable the prevention and suppression of certain behaviors related to acts of corruption in the sector. - Identification of issues related to the practical application of these provisions in view of the fight against this phenomenon. - Identification of relevant suggestions to increase the effectiveness of anti-corruption measures and mechanisms to control and fight against corruption in public administration.
\end{abstract}

Keywords: Public administration, Public functionary, Senior state officials and local elected, Corruption, Active corruption, Criminal Code, Illegal Influence

\section{Introduzione}

Transparency International considera il 2011 come l'anno della crisi nel governare, mentre rileva che le proteste in tutto il mondo sono un indice significativo dell'indignazione contro la corruzione in politica e nel settore pubblico, esortate dalla mancanza della stabilita' economica e dalla evidente concezione dei cittadini della mancanza della trasparenza dei leader e delle istituzioni pubbliche ${ }^{1}$.

Nonostante gli studi ed i sondaggi testimoniano di un miglioramento della situazione ${ }^{2}$, la corruzione continua ad essere un fenomeno preoccupante per la societa' albanese ${ }^{3}$. La necessita' di indirizzare la lotta alla corruzione

\footnotetext{
1 Transparency Interantional Albania, rapporto del $1^{\circ}$ dicembre 2011, pg. 1 (www.tia.al).

${ }^{2}$ Dai sondaggi effettuati da Transparency International, l'Albania e' scesa di 8 posti nella classifica rispetto all'anno 2010. Per maggiori informazioni v. Transparency International, Corruption Perception Index 2011, Interantional Secretariat, pg. 3 (www.transparency.org). 3 /l Progress Rapporto della Comunita' Europea per l'Albania considera che la corruzione e' diffusa in molti settori e costituisce un problema particolarmente grave. Per maggiori informazioni v. Albania 2011 Progress Report, Commission staff working paper, Brussels, 12.10.2011, sec(2011) 1205 final, parte Politiche Anticorruzione, pg 16.
} 
costituisce una delle principali questioni di interesse pubblico. La corruzione nel settore della pubblica amministrazione e' uno dei fenomeni su cui la percezione dei cittadini continua ad essere alta 4 .

Tale forma di corruzione non è un fenomeno nuovo per la societa' albanese. La corruzione nel settore pubblico è stata parte dell'attivita' sociale sin da tempi remoti. Dal punto di vista storico, dagli studi si evince che tale fenomeno risale nel periodo dell'invasione ottomana. II riferimento a tale periodo storico si basa sull'etimologia turca delle parole utilizzate nella vita quotidiana per descrivere il fenomeno della corruzione quali "bakshish", "ryshfet" oppure "qelepir"s.

Durante il regime comunista, la forma rigorosa del sistema costituiva una barriera per la corruzione. Comunque, dagli studi effettuati su tale periodo, si evince che la corruzione era una caratteristica del sistema comunista. In particolare si considera che la corruzione diviene un fenomeno assai diffuso negli anni ' 80 , in particolare nel settore occupazionale, delle nomine e in quello delle assegnazioni delle borse di studio universitarie ${ }^{6}$. In tale periodo le forme di corruzione più che alla ricezione della tangente "ryshfet" si riferivano ai favoreggiamenti in base ai rapporti di amicizia o di clientelismo.

Dopo gli anni '90, in particolare dopo il fallimento delle finanziarie, la corruzione ha riconosciuto immense dimensioni. Nel corso degli anni 2000, tranne qualche piccola oscillazione, l'indice della corruzione continua ad essere alto $^{7}$. Dai sondaggi effettuati in questo periodo risulta anche una alta percezione dei cittadini riguardo alla corruzione ${ }^{8}$.

Una delle principali caratteristiche della corruzione nel periodo della democrazia e' la manifestazione di nuove forme. Piu' che uno strumento per procurarsi dei vantaggi in base ai rapporti sociali essa è considerata come un mezzo per ottenere vantaggi personali e la forma piu' diffusa e' la concussione o la ricezione di "ryshfef" (tangente), in cambio di favori. Oltre alla dominante dimensione economica, cioe' il dare e ricevere "ryshfet" (tangente), anche quella sociale continua ad essere molto diffusa. Nel contesto albanese, dove le pratiche di corruzione quali il nepotismo, cioe' l'ottenimento dei vantaggi in base ai rapporti familiari o di parentela, o il clientelismo secondo cui i vantaggi si procurano in base alla fedelta' 0 all'appartenenza politica, sono piu' che presenti, tale dimensione assume una particolare importanza 9 .

Sono varie le definizioni del termine corruzione, però quella piu' classica la definisce come "un abuso di potere pubblico a fini privati" 10 mettendo l'accento sulla corruzione nel settore pubblico quale forma più diffusa della corruzione. Con riferimento a tale definizione gli studiosi sono del parere che la corruzione include la concussione (ryshfet), l'estorsione, l'appropriazione indebita, il nepotismo, l'utilizzo di beni pubblici a fini personali ed il traffico di inflenze illecite11.

Una definizione giuridica della corruzione si trova nella Convenzione Civile "Sulla Corruzione" ratificata dallo Stato albanese con la Legge nr. 8365 del 06/07.2000. Ai sensi dell'art.2 della presente Convenzione la corruzione assume il significato di "richiedere, offrire, dare 0 accettare direttamente 0 indirettamente del denaro od altra utilita' 0 qualsiasi altro

\footnotetext{
${ }^{4}$ Da un sondaggio del 2009, 48,5\% degli intervistati ritengono che il livello di corruzione dei funzionari pubblici e' aumentato rispetto all'anno precedente. Solo il $13,5 \%$ degli intervistati ritengono che il livello di corruzione sia diminuito. Per maggiori informazioni v. USAID, Corruzione in Albania, Percezioni ed esperienze, Institute for Development Research and alternatives, (IDRA) 2009, .(http://idraal.org/es2009coruption\%20inAlbania\%2009\%20\%20Summary\%20Findings.pdf), pg 12. La stessa percezione, che la corruzione dei funzionari pubblici sia molto diffusa, risulta anche dai sondaggi effettuati nel 2010. Per maggiori informazioni v. USAID Corruption in Albania 2010, (http://albania.usaid.gov/shfaqart/465/26/Corruption in albania2010survey.htm).

${ }^{5}$ Swedish Internatioanl Devepopment Cooperation Agency (SIDA), Albanian Anticoruption study, Ramboll Manegement, 2008, pg 7, (http://www.aidharmonisation.org.al/skedarët/1203101753-SIDA\%Albania\%Anti-Corruption\%Study\%20-\%Revise.pdf).

6 Ivi, pg. 7

${ }^{7}$ Dai sondaggi si evince che i dati sulla corruzione in Albania sono quasi stessi, circa 2,5 nel periodo 2002 - 2005, con un leggero calo nel 2005 (2,4), incrementato nel 2006 (2,6) e nel 2007 (2,9). Per maggiori informazioni v. Transparency International Corruption percepton Index, 2009 (htttp://www.transparency.org/policy_research/surve_indices/cp/2009) e Sida, Albanian Anticoruption study, opera citata, pg 54.

${ }^{8}$ Dai sondaggi dell'IDRA risulta che nel 2006 50\% degli intervistati hanno vissuto un'esperienza di corruzione. Per quanto riguarda il 2009, quasi la meta' degli intervistati (48,5\%) ritiene che la corruzione e' incrementata rispetto all'anno precedente, mentre il $38 \%$ ritiene che e' rimasta invariata. Per maggiori informazioni v. (DRA) survey, 2009, opera citata, pg 3.

${ }^{9}$ Gli studi effettuati testimoniano che la societa' albanese spesso reagisce in base ai rapporti ed alle relazioni personali e non in base al professionalismo ed alla trasparenza. Per maggiori informazioni v. Daniela Irrera, The balkanisation of politics, crime and coruption in Albania, Europian University Institutee, EUI Working Paper, RSCAS 2006, pg 10.

10 Definizione della Banca Mondiale, pagina ufficiale www.worldbanc.com.

11 U, Mynit, Corruption, Causes, consequences and cures, Asia Pcific Deveplopmet Juornal, Vol 7, No.2, December 2000, pg 35 (http://www.unescap.org/DRPAD/publication/juornal-7-2myint.pdf).
} 
vantaggio che fanno deviare il pubblico ufficiale dall'esercizio del dovere procurandosi di conseguenza del vantaggio indebito".

Come si nota dalla definizione dal punto di vista giuridico - penale la corruzione può essere considerata come un'azione criminale che garantisce ai soggetti coinvolti dupplici vantaggi.

Da una parte il soggetto che corrompe tende a realizzare un diritto 0 un'utilita' a favore dei propri interessi dando 0 promettendo qualsiasi indebito profitto, mentre il soggetto che viene corrotto si procura dei vantaggi, assicurando in compenso dei servizi favorevoli, abusando illecitamente delle proprie competenze o funzioni.

Nonostante gli studi ed i sondaggi effettuati sulla corruzione, è difficile avere una visione chiara delle dimensioni e della diffusione del fenomeno. La difficolta' consiste nella percezione dei cittadini sulla corruzione che spesso non corrisponde a quella giuridica. Questa difficolta' e' stata evidenziata anche dagli organismi che hanno effettuato i sondaggi sulla diffusione della corruzione e sul livello di percezione della stessa. Dai sondaggi e' emmerso che i cittadini considerano come atto di corruzione anche quei casi che non corrispondono al significato della corruzione, come per esempio l'aumento del prezzo dei fiori da parte dei fiorai nei giorni di festa ${ }^{12}$. Si fa confusione anche con la terminologia della corruzione e la linea di demarcazione tra le parole "bribe" (ryshfet) e "tip" (bakshish) che spesso vengono utilizzate con lo stesso significato nonostante siano dei concetti diversi ${ }^{13}$. Gli stessi sondaggi dimostrano che per quanto riguarda le parti coinvolte nella corruzione, gli albanesi sono piu' tolleranti verso i concussi che verso i concussori ${ }^{14}$.

Dopo il 2007 sono stati intrapresi dei passi positivi che miravano a frenare la corruzione in settori come il servizio civile, le gare d'appalto ecc. Si presume che cio' abbia inciso sul calo del numero delle esperienze di corruzione da parte del pubblico ${ }^{15}$. Facendo riferimento all'anno 2011, l'Albania viene classificata al 95simo posto tra i 183 Paesi coinvolti, aggiudicata con 3.1 punti, registrando cosi' un leggero miglioramento rispetto al $2010^{16}$.

Tale situazione è risultato degli impegni assunti al contrasto della corruzione in tutte le sue forme e dimensioni e certamente un ruolo principale ha avuto anche la legislazione penale e la riforma di legge in generale.

Obiettivo di questa relazione è la presentazione delle questioni inerenti la riforma legislativa anticorruzione e la loro efficacia al contrasto della corruzione nella pubblica ammnistrazione.

\section{II Quadro Storico dei Reati nell'ambito della Corruzione}

\subsection{Ovvero il Trattamento giuridico - penale dal punto di vista storico}

Il Codice Penale del Re Zog offre un trattamento giuridico dei reati riguardo a corruzione nel settore pubblico 17. La categoria di reati simili è prevista dal terzo Capitolo, dagli artt. 192 - 199. Sotto il profilo giuridico, la corruzione si identificata con il termine "concussione" ed e' previsto sia la dazione che la ricezione. Nel caso della concussione soggetto della responsibilita' penale è il pubblico ufficiale che procura per se' vantaggi che non dovuti. La concussione è punibile sia nel caso in cui il pubblico ufficiale compie un atto del suo ufficio che nel caso in cui compie un atto contrario ai doveri d'ufficio. Le disposizioni offrono un trattamento dettagliato e sono caratterizzate da un rigoroso sistema

\footnotetext{
12. Conclusione evidenziata da un sondaggio dell'IDRA ove il 25\% degli intervistati l'hanno considerato come un atto di corruzione che deve essere punito. Per maggiori informazioni v. IDRA survey, opera citata, pg. 2, e Sida, Albanian Anticoruption study,2008, opera citata, pg 58.

${ }_{13}^{13}$ Sida, Albanian Anticoruption study,2008, opera citata, pg 58.

${ }^{14}$ Cosi' per esempio un allievo che da' all'insegnante una camicia nella speranza di ottenere un voto alto viene considerato non corrotto (35,4\%) o giustificato (34,7\%). Per maggiori informazioni v. IDRA survey 2009, opera citata, pg. 4.

${ }^{15}$ Dai sondaggi si evince che il numero degli intervistati che hanno avuto un'esperienza di corruzione nel corso del 2009 e' circa 5\% piu' basso rispetto al 2008 e circa 9\% piu' basso rispetto al 2005. Per maggiori informazioni v. IDRA survey 2009, opera citata, pg. 19.

${ }^{16}$ Bazuar në Indeksin e Perceptimit të Korrupsionit për vitin 2011 realizuar për 183 vende, Shqipëria shënon 3.1 pikë, nga 3.3 që ishte për vitin 2010. në këtë mënyrë Shqipëria renditet në vendin e 95, krahasuar me vendin e 87 në vitin paraardhës, duke zbritur në klasifikim 8 vende më poshtë se në 2010. Për më shumë shih, Transparency International, Corruption Perception Index 2011, vepër e cituar, fq 3. Basandosi sull'Indice di Percezione della Corruzione per l'anno 2011 realizzato in 183 Paesi, l'Albania viene aggiudicata con 3.1 punti rispetto a 3.3 nel 2010. Cosi' l'Albania viene classificata al 95simo posto. Per maggiori informazioni v. Transparency International, Corruption Perception Index 2011, opera citata, pg 3.

${ }^{17}$ Ky Kod hyri në fuqi me në vitin 1927 me shkresën nr. 83-1 datë 28.05. 1927 të Kryesisë së Senatit. I/ Codice e' entrato in vigore nel 1927 con nota $\mathrm{nr}$. 83-1 del 28/05/1927 della Presidenza del Senato.
} 
sanzionatorio18. In tutti i casi sono previste le pene reclusorie e quelle pecuniarie. In caso di concussione, considerato che soggetto della responsabilita' è un pubblico ufficiale, alla categoria delle sanzioni si aggiunge anche l'interdizione dai pubblici uffici. La dazione è prevista dall'art. 196 del Codice Penale secondo cui ogni persona che offre 0 fa da tramite nell'offrire un'utilità ad un pubblico ufficiale, soggiace alla responsabilita' penale.

Dopo la liberazione, in Albania si stabilisce una nuova forma di regime basata sulla dittatura comunista. II nuovo Codice Penale 19 si caratterizza dalla severita' e dall'ideologizzazione, caratteristiche queste presenti anche nel trattamento della categoria dei reati riguardanti la corruzione. Tale categoria è trattata dal Capitolo VIII sui "Reati nell'esercizio del pubblico impiego". I reati di corruzione sono previsti da soli due articoli del Codice Penale, dall'art. 204 e dall'art. 205 che rispettivamente stabiliscono il reato di dazione e di concussione. Anche in tal caso la terminologia giuridica prevede lo stesso termine "concussione" oppure "ryshfet". L'attivita' di concussione si caratterizza dal fatto che si compie da un pubblico ufficiale, addirittura si considera una circostanza specifica la ricezione dalle persone che coprono ruoli di particolare responsabilita'20. Soggetto della responsabilita' penale è il pubblico ufficiale 21.

Nel caso della dazione chiunque offre un'utilita' ad un pubblico ufficiale ai fini della commissione o della omissione di un atto d'ufficio o fa da tramite, e' soggetto della responsabilita' penale. Un'altra caratteristica della disposizione è l'esclusione dalla responsibilita' penale nel caso l'atto di ricezione si realizza dietro la costrinzione del pubblico ufficiale, quando manca quindi la volonta' di dazione, nonche' nei casi di volontaria denuncia da parte del concusso presso gli organi di giustizia22.

Nel 1977, anno in cui è entrato in vigore il nuovo Codice Penale 23 , il regime totalitario aveva gia' consolidato la sua posizione. II nuovo Codice non presenta alcuna sostanziale novità di contenuto, però i caratterizza da un profondo spirito ideologico e politico rispetto a quello precedente.

Tale tendenza di politicizzazione si nota nella nuova terminologia dei reati di carattere economico. Cosi' il termine "patrimonio statale e sociale" è sostituito dal termine "patrimonio socialista", a testimonianza del carattere ideologico e della posizione giuridica contro la proprieta' privata che e' quasi inesistente.24 Riguardo ai reati di corruzione nel settore pubblico non si verificano sostanziali modifiche.

Il reato di concussione è previsto da un'unica disposizione che stabilisce delle sanzioni rigorose 25 . Per quanto riguarda la formulazione, si nota uno stile più conciso nella redazione delle disposizioni.

Dopo la caduta del regime comunista, i cambiamenti democratici hanno imposto la necessita' della rivisione della legislazione, compresa quella penale. Gia' prima dell'approvazione del nuovo Codice Penale, si sono registrati degli sforzi di carattere normativo al fine di far corrispondere i reati di corruzione con la nuova realta'26.

L'approvazione del nuovo Codice Penale 27 costituisce un importante passo nell'ambito delle modifiche normative. II Codice, nel rispetto dei principi democratici, presenta un nuovo spirito nell'esaminare i reati. Nella fase iniziale, prima dei successivi emendamenti, il Codice non presenta alcuna sostanziale novita' rispetto al contenuto delle disposizioni nell'ambito della corruzione nel settore pubblico.

\footnotetext{
${ }^{18}$ Në disa raste për mitëmarjen parashikohej dënimi me burgim të rëndë jo më pak se 15 vjet. Për më shumë shih, neni 194/4 të Kodit Penal të Mbretërisë.

In alcuni casi contro la concussione era prevista la pena reclusoria non inferiore a 15 anni. Per maggiori informazioni v. Art. 194/4 del Codice Penale Regio.

${ }^{19}$ Approvato con la Legge nr. 470 del 23/05/1952.

20 Ai sensi dell'art. 204/2 del codice Penale del 1952: La concussione, o piu' di una volta, oppure da persona di particolare responsabilita' pubblica, e' punibile:con la reclusione fino a 10 anni.

${ }^{21}$ Onde evitare qualsiasi problema nella concreta attuazione delle disposizioni di cui sopra, l'art. 198 del Codice Penale stabilisce la definizione del pubblico ufficiale quale "persona che svolge funzioni permanenti o temporanee presso istituzioni ed enti statali, oppure presso organizzazioni sociali, per legge incaricati ad esercitare mansioni, diritti o competenze ai fini della realizzazione degli atti di carattere economico, professionale o di altri atti di carattere sociale".

22 Il Codice Penale dell'anno 1952, art. 205/2.

${ }^{23}$ Approvato con la Legge nr. 5591 del 15.06.1977.

${ }^{24}$ Capitolo II, "Reati contro il patrimonio socialista" N 61-68.

${ }^{25}$ Codice Penale dell'anno 1977, art. 109.

${ }^{26}$ Prima dell'approvazione del Codice Penale nel 1995, le modifiche nell'ambito penale contro la corruzione sono state apportate con la Legge nr. 7533 del 30/01/1992. Con questa Legge nel Codice Penale del 1977 e' stato inserito l'art. 60/a che stabilisce la corruzione politica. La pena prevista e' quella detentiva non inferiore a dieci anni di reclusione. Per maggiori informazioni v. I.Elezi (1998), Lo sviluppo storico della legislazione penale in Albania, albin, Tirana, pg. 119.

${ }^{27}$ Approvato con la Legge nr. 7895 del 27/01/1995.
} 


\subsection{Gli emendamenti del Codice Penale e l'importanza del contrasto alla corruzione nel settore pubblico}

Gli emendamenti del Codice Penale corrispondono all'esigenza di adempimento degli impegni internazionali ed all'avvicinamento della legislazione agli standard internazionali.

Nell'adempiere gli impegni internazionali assunti, lo Stato albanese ha ratificato una serie di strumenti internazionali del settore facendosi parte anche delle iniziative internazionali anticorruzione. Negli anni $2000-2001$, l'Albania ha ratificato la Convenzione civile "Sulla Corruzione" 28 , la Convenzione penale "Sulla Corruzione" 29 del Consiglio d'Europa, che comporta degli aspetti importanti per la riforma della legislazione penale ${ }^{30}$ ed il relativo protocollo aggiuntivo e in seguito la Convenzione delle Nazioni Unite "Sulla Corruzione" "31.

L'Albania, dopo i cambiamenti democratici, è diventata parte degli sviluppi internazionali e regionali nell'ambito della lotta al fenomeno della corruzione, è già membro di GRECO (Gruppo degli Stati Contro la Corruzione del Consiglio d'Europa), parte dell'iniziativa SPAI (Iniziativa Anticorruzione del Patto di Stabilita). Inoltre è coinvolta in una serie di programmi contro corruzione dei Paesi dell'Europa Sud Orientale (PACO) e di altri programmi messi in atto dagli organismi internazionali che operano in Albania.

In considerazione degli impegni assunti, il nuovo Codice Penale nell'ambito dei reati di corruzione ha subito una serie di modifiche affinchè la formulazione della corruzione sia in linea con gli atti giuridici internazionali ${ }^{32}$. Nell'ambito dei reati di corruzione, il Codice Penale e' stato emendato con la Legge nr. 9086 del 19/06/2003, con la Legge nr. 9275 del 16/09/2004 e con la Legge nr. 9686 del 26/02/2007.

Attraverso tali emendamenti e' stato perfezionato il contenuto e la presentazione dettagliata delle disposizioni e sono state irrigidite le sanzioni. E' stata modificata anche la terminologia, cosi' i termini "concussione" e "dazione" sono stati sostituiti dai termini "corruzione attiva" e "corruzione passiva".

II Codice Penale si è arricchito inoltre di nuove disposizioni al fine del contrasto degli atti di corruzione nel settore pubblico. In concreto, il Codice Penale prevede nuove disposizioni inerenti la corruzione attiva e passiva degli alti funzionari dello stato e degli eletti locali, reati questi non previsti dal precedente Codice. Questo nuovo modo di riflessione corrisponde ai requisiti della Convenzione penale "Contro la corruzione" che impone agli stati membri la previsione di nuove forme di corruzione in risposta alle nuove forme e tendenze di manifestazione della stessa.

Seguirà un'analisi dettagliata delle disposizioni sopra citate.

Con la Legge nr. 9275 del 16/09/2004 e' stato perfezionato il contenuto delle vigenti disposizioni del Codice Penale al contrasto della corruzione nel settore pubblico, come per esempio il contenuto degli artt. 244 e 259 del Codice Penale che stabilisce la corruzione attiva e la corruzione passiva delle persone che esercitano funzioni pubbliche ${ }^{33}$. Tale forma di corruzione è tipica dell'abuso d'ufficio, quando un pubblico ufficiale 0 un incaricato di pubblico servizio, approfittando della posizione che riveste, procura a se' un indebito vantaggio patrimoniale o non patrimoniale. Tale reato si manifesta sotto forma di richiesta, ricezione 0 accettazione di un indebito profitto dalla persona che esercita funzioni pubbliche. Nella vita quotidiana con il termine "indebito profitto" si sottintende non solo il proffitto di denaro, ma anche di merci, di doni o di qualsiasi altro servizio a favore. Nel concreto un simile reato si verifica nel momento in cui il medico richiede 0 accetta un indebito vantaggio in cambio della cura del paziente, oppure mentre l'ufficiale dello stato civile richiede un indebito vantaggio in cambio del rilascio del certificato o di qualsiasi altro documento.

A queste attivita' criminali che tendono a procurare a sè 0 ad altri un indebito vantaggio corrisponde il sistema delle

\footnotetext{
${ }^{28}$ Legge nr. 8635 del 06/07/2000 per la ratifica della Convenzione civile "Sulla corruzione".

${ }^{29}$ Legge nr. 8778 del 26/04/2001 per la ratifica della Convenzione penale "Sulla Corruzione", modificato.

30 V. Hysi (2007), "La politica penale albanese nell'ambito dei reati di corruzione”, Studi giuridici, nr. 1, Pegi, Tirana, pg 41.

31 Legge nr. 9492 del 13/03/2006 per la ratifica della Convenzione delle Nazioni Unite "Contro la corruzione".

32 La Convenzione penale "Sulla corruzione“ impone agli stati membri una serie di provvedimenti legislativi che nella maggior parte sono stati riportati nella nuova legislazione penale.

${ }^{33}$ Art. 259 del Codice Penale, emendato con la Legge nr. 9275 del 16/09/2004, prevede la seguente formula per corruzione passiva delle persone che esercitano funzioni pubbliche: "Kërkimi ose marrja drejtpërdrejt ose tërthorazi, I cfardo përfitimi të parregullt apo i një premtimi të tillë, për vete ose për persona të tjerë, ose pranimi i një oferte apo premtimi që vjen nga përfitimi i parregullt nga personi që ushtron funksion publik, për të kryer ose moskryer një veprim që lidhet me detyrën apo funksionin e tij dënohet me burgim nga dy deri në tetë vjet dhe me gjobë nga pesëqindmijë deri në tre milion lekë". "La richiesta o la ricezione, direttamente o indirettamente, di un indebito vantaggio o di una simile promessa, per se' o per un terzo, oppure l'accettazione di un'offerta o di una promessa ingiusta da un pubblico ufficiale per compiere o omettere un atto del suo ufficio e' punita con la reclusione da due a otto anni e con la multa da cinquecentomila a tre milioni di Lek".
} 
sanzioni stabilite per questa categoria di reati, che prevede non solo la pena detentiva ma anche quella pecuniaria. ${ }^{34}$

Un aspetto importante delle modifiche apportate è anche l'ampliamento dei soggetti sottoposti alla responsabilita' penale che si estende agli alti funzionari dello stato ed agli eletti locali. Ciò a seguito delle nuove forme di corruzione, come la corruzione attiva e passiva degli alti funzionari dello stato e degli eletti locali (artt. 245 e 260) ${ }^{35}$. In tal modo è possibile punire gli atti di corruzione degli alti funzionari della pubblica aministrazione e degli eletti locali, quali sindaci ecc.

II Codice Penale presenta un'altra novita', l'estensione della responsabilita' penale alle persone che illecitamente inducono un pubblico ufficiale a compiere un atto d'ufficio. In tal modo viene punito il "traffico delle influenze", che nella terminologia giuridica viene denominata "traffico illecito", inserendo l'art. 245/a ${ }^{36}$ che prevede proprio questa forma di corruzione. Tale modifica corrisponde all'art. 12 della convenzione penale "Contro la corruzione" che impone la previsione dalla legislazione penale nazionale delle forme dell'induzione illecita. La formulazione della disposizione corrisponde esattamente alle forme ed ai meccanismi soffisticati, talvolta indiretti, delle pratiche di corruzione. In realta' non sono pochi i casi in cui queste persone, normali cittadini o pubblici ufficiali essi siano, fanno uso delle proprie capacita' ad influenzare illecitamente nella presa delle decisioni nel corso dell'esercizio delle funzioni pubbliche.

E' molto positivo il fatto delll'estensione della responsabilita' penale, nonostante l'avvenuta realizzazione dell'atto criminale o dell'esercizio dell'influenza illecita. Per l'applicazione della responsabilita' penale è sufficiente la verifica di una sola forma, la promessa, la proposta o la concessione di un indebito vantaggio, nonche' la richiesta, la ricezione 0 l'accettazione dello stesso.

La manifestazione di nuove forme di corruzione ed il contrasto a tale attivita' criminale comporta la necessita' dell'aumento della consapevolezza cittadina a denunciare i casi di corruzione ed a collaborare con gli organi di giustizia. Percio', ai sensi dell'art. 26 del Codice Penale, è prevista la riduzione della pena o l'esclusione dalla responsabilita' penale per chi presenta denuncia o collabora nel procedimento penale degli atti di corruzione (art. 245/2). ${ }^{37}$.

Con la presente disposizione si tende ad incoraggiare tutti i cittadini che hanno partecipato ad attivita' di corruzione dando o promettendo vantaggi od altre utilita' a denunciare gli atti di corruzione, portando al procedimento penale di questi atti. Tale formulazione tende a scoprire gli atti di corruzione e gli autori. Al fine di incoraggiare i cittadini a presentare denuncia in tempi reali e prima dell'avvenuta conseguenza criminale, l'ultimo comma stabilisce che il tribunale nel pronunciare la sentenza deve tenere presente anche il momento in cui è stata presentata la denuncia nonche' l'avvenuta verifica delle conseguenze. Con riferimento a tali fattori il tribunale puo' disporre la riduzione della pena sotto il minimo previsto dalla legge oppure l'esclusione dalla responsabilita' penale.

Anche se non direttamente connessa al reato di corruzione, la previsione come reato della condotta di occultamento e riciclaggio del prodotto criminale contribuisce al contrasto della corruzione stessa. Tale disposizione, prima denominata "alienazione del bene", e' stata modificata con la Legge nr. 9086 del 19.06.2003. Ora è previsto dall'art. 287 del Codice Penale. La nuova disposizione è stata formulata in modo dettagliato e prevede tutte le forme di riciclaggio di denaro di provenienza delittuosa che tendono ad occultare l'origine, la natura, o la fonte del bene, prodotto del reato.

Un'altra forma di occultamento del bene sanzionata dal Codice Penale e' anche "l'apertura di conti correnti anonimi", previsto dall'art. 287/a, che stabilisce delle sanzioni per l'apertura dei conti correnti anonimi 0 a favore di

\footnotetext{
${ }^{34}$ Sarebbe opportuno che tale sistema di sanzioni sia unificato per tutti i tipi del reato della corruzione.

${ }^{35}$ La formulazione della disposizione e' simile a quella della corruzione passiva degli alti funzionari dello stato. La distinzione consiste nel soggetto della responsabilita' penale, che nel caso concreto e' un alto funzionario dello stato oppure un eletto locale.

${ }^{36}$ Nella Legge nr. 9275 del 16/09/2004 si inserisce l'art. 245/a che stabilisce quanto segue: "La promessa, l'offerta o la dazione, direttamente o indirettamente, di qualsiasi indebito vantaggio, a se' o a terzi, alla persona che promette o garantisce di essere in grado di esercitare illecita influenza sulle persone incaricate ad esercitare funzioni pubbliche per compire un atto d'ufficio o per prendere una decisione, albanesi o stranieri essi siano, nonostante l'avvenuta influenza o conseguenza, costituisce reato ed e' punito con la reclusione da sei mesi a due anni e con la multa da trecentomila a un milione di Leke. La richiesta, la ricezione o l'accettazione, direttamente o indirettamente, di qualsiasi indebito vantaggio, a se' o a terzi, con la promessa di esercitare illecita influenza sui pubblici ufficiali per compiere un atto d'ufficio, albanesi o stranieri essi siano, nonostante l'avvenuta influenza o conseguenza, costituisce reato ed e' punito con la reclusione da sei mesi a quattro anni e con la multa da cinquecentomila a due milioni di Leke".

${ }^{37}$ Ai sensi dell'art. 245, modificato con la Legge nr. 9275 del 16/09/2004 : "Colui che promette oppure dà del denaro o altre utilità, ai sensi degli artt. 164/a, 244, 245, 312, 319 e 328 del presente Codice, può beneficiare dell'esclusione dalla responsabilita' penale o la riduzione della pena, secondo quanto previsto dall'art. 28 del Codice, se presenta denuncia o collabora al procedimento penale di tali atti. Nell'emettere la sentenza, il tribunale deve tenere presente il periodo in cui e' stata presentata la denuncia e l'avvenuta verifica delle conseguenze".
} 
prestanomi. Tale disposizione tende inoltre alla prevenzione del riciclaggio di denaro, prodotto di attivita' delittuose. L'approvazione della Legge nr. 8610 del 07/05/2000 "Sulla prevenzione del riciclaggio di denaro" ha reso necessario l'inserimento nel Codice Penale di una simile disposizione.

L'inserimento delle disposizioni di cui sopra alla categoria dei reati contro l'ordine e la sicurezza pubblica, tende a contrastare l'attivita' criminale ad alto rischio sociale quali il traffico delle sostanze stupefacenti, il traffico d'armi e di munizioni, ecc. Tali disposizioni sono degli utili strumenti al contrasto del reato di corruzione, in particolare alla scoperta dei beni 0 di qualsiasi altro provente criminale. Va sottolineato il fatto che la Convenzione penale sulla corruzione connette le attivita' di riciclaggio dei proventi del reato con gli atti di corruzione ${ }^{38}$.

Parte delle pratiche di corruzione sono anche le forme di abuso di potere previste dal Codice Penale quali per esempio, "l'abuso con i finanziamenti dello stato"39, oppure "la violazione del principio di equita' dei partecipanti alle gare 0 appalti pubblici"40. Nell'ambito della riforma del Codice Penale queste disposizioni dovevano subire delle modifiche necessarie sia per l'unificazione della terminologia in armonia con le altre disposizioni che per la modifica delle sanzioni. Ora sono state intraprese delle iniziative legislative volte ad effettuare ulteriori emendamenti al Codice Penale, prevedendo nuove forme di corruzione come - la corruzione attiva e passiva dei pubblici agenti stranieri e dei funzionari degli organismi internazionali, l'arricchimento illecito, ecc. al fine di corrispondere pienamente agli atti internazionali in ambito della corruzione. Fino adesso tali proposte di legge non sono ancora state materializzate in pratica.

\subsection{Altri provvedimenti giuridici anticorruzione nel settore della pubblica amministrazione}

Oltre agli atti aggiuntivi ed alle modifiche apportate al Codice Penale, il contrasto alla corruzione ha trovato applicazione anche nell'approvazione della specifica legge anti-corruzione. L'obiettivo della legislazione è la sanzione dei doveri, dei divieti e delle procedure atte alla prevenzione ed al contrasto di condotte di corruzione nel settore pubblico. In tale ambito vale menzionare:

- la legge per le gare pubbliche, grazie alle nuove procedure, aumenta il livello di trasparenza delle gare d'appalto prevenendo cosi' gli eventuali atti di corruzione nel processo di gara;

- la legge che garantisce la collaborazione del pubblico al contrasto della corruzione ${ }^{41}$;

- la legge che vieta i conflitti d'interesse nell'esercizio delle funzioni pubbliche ${ }^{42}$;

- la legge che disciplina le responsabilità ed i doveri dei pubblici ufficiali nel dichiarare i redditi ed i beni 43.

Considerata la particolare importanza, ci fermiamo brevemente ad analizzare la legge sulla dichiarazione ed il controllo dei beni. La legge ha subito una serie di modifiche atte a migliorare il contenuto ed il livello di attuazione della stessa $^{44}$. L'obiettivo della legge e' quello di stabilire delle norme per la dichiarazione ed il controllo dei beni e la legittimita' delle fonti dei funzionari pubblici ${ }^{45}$. Al fine di garantire la trasparenza dei proventi finanziari, la legge sanziona l'obbligo per i funzionari pubblici a dichiarare i redditi ed i loro beni. La legge stabilisce un vasto numero di soggetti che soggiaciono all'obbligo di dichiarare i beni, a partire dagli Alti Funzionari dello Stato, ivi incluso il Presidente della Repubblica, i deputati del Parlamento, il Primo Ministro, il Vice Primo Ministro, i ministri ed i vice ministri, i funzionari degli organi amministrativi, ecc. e tutti gli impiegati della pubblica amministrazione. Riguardo agli impiegati della pubblica amministrazione, l'obbligo di dichiarazione si riferisce a quella categoria di impiegati che controllano e gestiscono fondi

\footnotetext{
${ }^{38}$ L'art. 13 della Convenzione Penale "Sulla corruzione" prevede l'obbligo per gli Stati aderenti ad intraprendere dei provvedimenti atti a stabilire in seno alle legislazioni interne il reato di "riciclaggio di denaro di provenienza dall'attivita' di corruzione".

${ }^{39}$ Codice Penale, art. 256.

${ }^{40}$ Codice Penale, art. 258.

41 La Legge nr. 9508 del 03/04.2006 "Sulla collaborazione del pubblico al contrasto della corruzione“.

42 La Legge nr. 9367 del 07/04/2005 "Sulla prevenzione del conflitto d'interesse nell'esercizio delle funzioni pubbliche", modificata.

${ }^{43}$ L'obbligo per la dichiarazione dei beni inizialmente era sanzionato con la Legge $\mathrm{nr}$. 7903 dell'8/03/1995 "Sulla dichiarazione di beni degli eletti e di alcuni funzionari e impiegati del pubblico servizio". Le lacune della legge hanno comportato una serie di problematiche nell'attuazione in pratica della stessa, percio' e' stata approvata la Legge nr. 9049 del 10/04/2003 "Sulla dichiarazione ed il controllo dei beni, degli obblighi finanziari degli eletti e di alcuni impiegati pubblici" modificata, tuttora in vigore.

44 L'obbligo per la dichiarazione dei beni inizialmente era sanzionato con la Legge nr. 7903 dell'8/03/1995 "Sulla dichiarazione di beni degli eletti e di alcuni funzionari e impiegati del pubblico servizio". Le lacune della legge hanno comportato una serie di problematiche nell'attuazione in pratica della stessa, percio' e' stata approvata la Legge nr. 9049 del 10/04/2003 "Sulla dichiarazione ed il controllo dei beni, degli obblighi finanziari degli eletti e di alcuni impiegati pubblici" modificata, tuttora in vigore.

${ }^{45}$ La Legge nr. 9049 del 10.04.2003 "Sulla dichiarazione ed il controllo dei beni, degli obblighi finanziari degli eletti e $i$ alcuni impiegati pubblici", art. 1.
} 
pubblici o comunque raccolgono proventi dello stato. All'obbligo di dichiarazione soggiaciono anche i rappresentanti degli organi di giustizia, i pubblici ministeri, i magistrati, i funzionari dell'ufficio esecutorio di tutti i livelli ${ }^{46}$.

A differenza della precedente legge tale obbligo si estende anche ai familiari ed alle persone vicine ai funzionari pubblici che sono tenuti a dichiarare i beni ogni volta che gli viene richiesto. Onde evitare qualsiasi inesattezza, la legge stabilisce anche i beni, gli obblighi finanziari e le fonti di provenienza dei beni oggetto della dichiarazione ${ }^{47}$.

Al fine dell'attuazione in pratica della legge, uno dei provvedimenti istituzionali piu' importanti è l'istituzione di un meccanismo sostenibile e permanente competente per la gestione del processo di controllo e di dichiarazione dei beni conformemente ai presupposti di legge : l'Alto Ispettorato di Dichiarazione e di Controllo dei Beni (AIDCB) ${ }^{48}$.

Nonostante gli obblighi di legge, fino al 2003 i casi della mancata o della falsa dichiarazione erano considerati e trattati come semplici contavvenzioni amministrative anziche' come violazioni penali. Percio' con le modifiche del 2003 nel Codice Penale e' stato inserito l'art. $257 / \mathrm{a}^{49}$ che stabilisce le responsabilita' penali in caso di rifiuto della dichiarazione, di mancata dichiarazione, di occultamento o di falsa dichiarazione dei beni delle persone elette 0 dei funzionari pubblici. Con le modifiche del 2007 e' aumentato il livello di pericolo sociale di questo reato. Grazie ai cambiamenti effettuati, l'occultamento o la falsa dichiarazione dei beni sono considerati reati e non contravvenzioni, di conseguenza sono state aumentate anche le rispettive sanzioni. In tal caso soggetto della responsabilita' penale e' il pubblico ufficiale che non dichiara i propri beni oppure rilascia una falsa dichiarazione. Al fine dell'implementazione in pratica, l'Alto Ispettorato per la Dichiarazione ed il Controllo dei Beni ha proposto le emende all'art. 257/a del Codice Penale inserendo tra i soggetti del reato anche le persone vicine all'ufficiale dichiarante ${ }^{50}$. Tale proposta non e' stata ancora inserita nel Codice Penale. Si ritiene che la sanzione della responsabilita' penale anche alle persone vicine al soggetto dichiarante costituisce un passo positivo a garanzia della trasparenza dei beni e sulle fonti di guadagno dei pubblici ufficiali onde evitare l'occulatemento dell'origine di questi beni e delle fonti di guadagno.

Strettamente legata alla presente Legge è anche quella nr. 9367 del 07.04.2005 "Sulla prevenzione dei conflitti d'interesse nell'esercizio delle funzioni pubbliche" che mira a garantire un potere decisionale imparziale, trasparente, nell'interesse del pubblico e della sua affidabilita' verso le istituzioni pubbliche attraverso la prevenzione del conflitto tra l'interesse pubblico e quello privato di un pubblico ufficiale nel corso dell'esercizio delle proprie funzioni51. II legame si verifica anche dal fatto che la principale autorita' responsabile dell'implementazione della legge e' l'AIDCB che tende a monitorare l'attuazione delle procedure per l'identificazione, la dichiarazione, l'esame, la soluzione e la punizione dei casi di conflitti d'interesse. Ai fini di una rigorosa implementazione della presente legge, sono stati compiuti degli sforzi volti ad aumentare la capacita' della pubblica ammministrazione per la soluzione del conflitto d'interesse mediante la redazione della base giuridica e la formazione dello staff come componente del piano d'applicazione della strategia anticorruzione ${ }^{52}$.

\footnotetext{
46 Ivi, art. 3.

${ }^{47}$ Ai sensi della Legge nr. 9049 del 10/04/2003 nr. 9049 del 10/04/2003 "Sulla dichiarazione ed il controllo dei beni e degli obblighi finanziari degli eletti e di alcuni funzionari pubblici", sono oggetto di dichiarazione: i beni immobili ed i diritti reali su di essi; i beni mobili iscritti nei registri pubblici; gli oggetti dal valore superiore ai 5.000 dollari americani; il valore delle azioni delle carte di valore e parti dei capitali in possesso; il valore delle liquidita', le situazioni in cash, i conti correnti, i depositi, i buoni del tesoro ed i prestiti in lek o in valuta estera; gli obblighi finanziari delle persone fisiche e giuridiche espressi in lek o in valua estera; $i$ reditti personali annuali a seguito dei salari, delle adesioni in commissioni o bordi o di qualsiasi altra attivita' redittizia; le licenze o le patenti che producono reditti.

${ }^{48}$ L'Alto Ispettorato di Dichiarazione e di Controllo dei Beni ha iniziato a funzionare in base alla Legge nr. 9049 del 10/04/2003 "Sulla dichiarazione ed il controllo dei beni, degli obblighi finanziari degli eletti e di alcuni funzionari pubblici".

${ }^{49}$ Modificato con la Legge nr. 9030 del 13.03.2003. Con la Legge nr. 9686 del 26/02/2007 e' stato modificato il secondo comma che prevede l'occultamento o la falsa dichiarazione del bene.

${ }^{50}$ La disposizione 257/a del Codice Penale "Il rifiuto alla dichiarazione, la non dichiarazione, l'occultamento o la falsa dichiarazione dei beni delle persone elette o dei pubblici ufficiali" non offriva soluzione ai casi di rifiuto delle persone vicine al soggetto dichiarante in quanto soggetto di tale reato e' solo il pubblico ufficiale dichiarante o non le persone ad esso legate. Vedi, Alto Ispettorato di Dichiarazione e di Controllo dei Beni, Rapporto Annuale dell'Ispettore Generale per l'anno 2009, pg. 4 (www.hidaa.gov.al clicato in data 01/12/2010).

51 Legge nr. 9367 del 07.04.2005 "Sulla prevenzione del conflitto d'interesse nell'esercizio delle funzioni pubbliche", art. 1.

52 La strategia intersettoriale per la prevenzione, il contrasto alla corruzione e la governance trasparente, Piano d'azione triennale 2011 - 2013, approvato nel corso della riunione del Gruppo Interministeriale del Lavoro in data 8 Giugno 2011, punto 6.
} 


\section{Conclusioni e Raccomandazioni}

Dopo i cambiamenti democratici, l'Albania è divenuta parte degli sviluppi internazionali e regionali ratificando una complessita' di strumenti internazionali nell'ambito della corruzione. In linea con questi sviluppi sono stati fatti gia' degli sforzi per completare tutta la base giuridica al fine di frenare il fenomeno della corruzione e punire gli atti di corruzione. Nell'ambito dei passi concreti contro il fenomeno della corruzione una fondamentale importanza riveste anche l'armonizzazione del quadro normativo con le convenzioni in ambito di corruzione, in particolare gli emendamenti della legislazione penale quale principale strumento al contrasto della corruzione. Con le modifiche apportate, i provvedimenti legislativi imposti dalle convenzioni internazionali sono stati riportati in gran parte dalla nuova legislazione penale. Cosi' sono state realizzate le priorita' di breve termine del governo albanese al rispetto del Piano nazionale per l'attuazione dell'Accordo di Stabilizzazione Associazione (ASA) ${ }^{53}$.

Nell'ambito di tali priorita', il Codice Penale ha subito una serie di modifiche e di miglioramenti, in particolare negli anni 2001, 2004 e 2007. Grazie a queste modifiche la formulazione delle disposizioni di legge ha riconosciuto dei perfezionamenti, mentre il Codice Penale si è arricchito di nuove forme di corruzione in linea alle tendenze di manifestazione della corruzione stessa. Nella lotta alla corruzione nel settore pubblico particolare importanza riveste anche la sanzione delle disposizioni come la corruzione attiva e passiva dei pubblici ufficiali, degli alti funzionari dello stato e degli eletti locali, l'esercizio dell'illecita influenza, la corruzione nel settore della giustizia, ecc.

La sanzione dell'obbligo di dichiarazione dei beni e degli obblighi finanziari dei pubblici ufficiali, costituisce un altro passo positivo per la prevenzione della corruzione all'interno della pubblica amministrazione.

Nonostante il quadro giuridico abbia riconosciuto notevoli miglioramenti, la lotta alla corruzione rimane tuttora una grande sfida per le autorita' dello stato e per tutta la societa' albanese in generale. Le pratiche della corruzione continuano ad essere presenti sia sul piano economico che su quello sociale.

La corruzione costituisce un ostacolo per una efficace soluzione delle problematiche dei cittadini e per la realizzazione dei diritti e dei loro legittimi interessi. Inoltre, la manifestazione delle pratiche di corruzione incide sul calo della fiducia dei cittadini verso l'attivita' dello Stato. Gli effetti sono ancora piu' negativi se la corruzione si verifica nel settore pubblico. Cio' perchè gli individui considerano lo stato quale meccanismo di garanzia dei loro legittimi interessi, e nel momento in cui tali interessi sono violati dagli stessi funzionari pubblici, la perdita del rispetto e della fiducia verso gli organi statali raggiunge i parametri massimali.

Sul piano sociale, un aspetto molto importante è anche la percezione dei cittadini sulla corruzione. La frequente manifestazione delle pratiche di corruzione fa sì che i cittadini la considerino un normale fenomeno. E' semplicemente considerato come un mezzo che rende possibile la tempestiva soluzione dei loro problemi. La corruzione viene percepita dai cittadini come un fenomeno vitale, presente in tutti gli aspetti della loro attivita' quotidiana ove spesso sono loro stessi a istigare e a generare la corruzione.

Anche se la maggior parte dei cittadini è stata vittima delle pratiche di corruzione, sono pochi coloro che hanno consapevolezza del fatto che la corruzione costituisce un'attivita' criminale a fini di lucro.

I beni procurati dagli atti di corruzione non sono altro che dei proventi criminali. Nella vita quotidiana si sta manifestando in modo ostile il riciclaggio di tali proventi, come tutti i proventi delle attivita' criminali, sotto varie forme di investimento.

Non mancano in realta' anche le altre forme di corruzione come il nepotismo, i rapporti di clientelismo, ivi incluso quello politico.

I motivi di questa situazione sono diversi. Le frequenti modifiche della legislazione in certi casi hanno prodotto delle difficolta' nell'implementazione in pratica della stessa. La riforma legislativa, nonostante il suo orientamento verso gli strumenti internazionali, non e' stata completa. Sono necessari degli interventi legislativi, specie in ambito della perfezione della legislazione sul conflitto d'interesse e la dichiarazione dei beni. Altri interventi sono necessari per l'incriminazione degli atti di corruzione non previsti dal Codice Penale. Cosi' il reato di corruzione degli agenti stranieri 0 dei rappresentanti delle organizzazioni internazionali non è previsto dal Codice Penale. Tuttora non ha trovato applicazione la proposta dell'estensione della responsabilita' penale sulle persone vicine al pubblico ufficiale nei casi di non dichiarazione o di falsa dichiarazione dei beni.

Ovvio che le riforme di legge sono strettamente collegate alla gestione del diritto penale. Nella vita quotidiana

${ }^{53}$ Consiglio dei Ministri, Piano Nazionale per l'attuazione dell'Accordo di Stabilizzazione Associazione, punto 1.1.6. La lotta alla corruzione, Giugno, Tirana 2006. 
spesso si verificano casi di una mancata rigorosa applicazione della legislazione penale dagli organi di giustizia. II ruolo attivo degli organi di giustizia nell'attuazione della legislazione rimane uno degli aspetti più importanti della lotta alla corruzione. Sono questi organi che devono dar vita e rendere efficace la legislazione penale. La procura, essendo l'organo in cui si fondano le basi del procedimento penale, assume una particolare importanza. Tale ruolo diventa più significativo nei procedimenti penali sulla corruzione considerata la difficolta' che rappresenta la punizione di questa categoria di reati.

Tale difficolta' si accentua anche dal limitato numero delle denuncie da parte dei cittadini dei casi di corruzione e dalle problematiche per la loro verifica ${ }^{54}$. Percio' è necessaria la formazione specializzata della polizia giudiziaria, dei pubblici ministeri e dei giudici sulla corruzione e sugli eventuali rapporti di connessione della stessa con altri reati. E' stata ottima l'opera svolta dalla Scuola di Magistratura in tal senso attraverso le continue attivita' di formazione dei giudici e dei pubblici ministeri. Anche le strutture di polizia hanno realizzato una serie di attivita' di formazione nell'ambito di progetti come PAMECA III, OPDAT, PACA, ecc ${ }^{55}$. Queste attivita' di formazione sono necessarie anche perche' le indagini dei casi di corruzione richiedono l'utilizzo di particolari tecniche di investigazione per l'acquisizione delle prove. La realizzazione di un'adeguata infrastruttura ed il rafforzamento della cooperazione tra gli organi di giustizia e gli altri organi statali sono un requisito necessario per la lotta alla corruzione.

La parzialita' politica nelle nomine e la mancanza delle garanzie per la stabilita' dell'impiego nella pubblica amministrazione rimane un pericolo di corruzione. Diventa fonte di irresponsabilita' e sollecita le condotte corruttive. Qundi, è necessario incoraggiare l'integrita' e l'onesta dei pubblici ufficiali attraverso la rigorosa attuazione della legge. Sono inoltre necessarie le modifiche da apportare alla Legge "Sullo status dell'impiegato pubblico", in particolare per quanto riguarda le procedure di assunzione e di promozione perche' l'integrita' e la professionalita' dei pubblici ufficiali sia ellevata ${ }^{56}$.

La piena immunita' degli alti funzionari rimane tuttora un ostacolo per le indagini dei casi di corruzione. Nonostante i tentativi per togliere l'immunita', il loro valore giuridico è molto discutibile. La sanzione dell'immunita' dalla Costituzione della Repubblica di Albania degli alti funzionari dello stato quali deputati, ministri ma anche magistrati, di conseguenza comporta la necessita' di apportare delle modifiche alla Costituzione ma fino adesso non c'e' stato alcun consenso tra le parti.

La corruzione non è semplicemente un fenomeno giuridico ma anche un fenomeno sociale. Quindi, in linea alle raccomandazioni di GRECO ${ }^{57}$ le misure di prevenzione non devono interessare solo il settore legale ma anche quello politico, sociale ed economico. Considerata la realta' albanese, la questione della corruzione richiede un impegno generale anche attraverso il sistema delle riforme sociali al fine di minimizzare gli atti di corruzione e di cambiare la condotta e la percezione della corruzione come un dannoso fenomeno che deve essere contrastato e non diventato uno stile di vita.

In tal senso i media sono un'arma potente per la sensibilizzazione e la crescita della consapevolezza cittadina.

In Albania, in genere, il ruolo dei media su tale fenomeno è stato molto positivo. Addirittura in alcuni casi i media sono stati gli iniziatori principali per l'avvio delle indagini, da parte dagli organi di giustizia, sugli atti di corruzione dei pubblici ufficiali. Tale percezione sul ruolo positivo dei media nella lotta alla corruzione e' stata evidenziata anche dal pubblico58.

Anche il ruolo della societa' civile e' determinante per la consocenza del fenomeno e la crescita della

54 "L'aumento dell'accesso dei cittadini agli organi di giustizia al fine di una efficacia attuazione della legislazione albanese, quale importante strumento alla lotta contro la corruzione". Pubblicazione del Centro per l'iniziativa legislativa e cittadina, Tirana, giugno 2007, pg. 18.

55 Queste attivita' di formazione sono iniziate nel 2011 e continueranno per tutto il periodo rimasto. Per maggiori informazioni v. il piano triennale d'azione 2011 - 2013 della Strategia intersettoriale per la prevenzione e la lotta alla corruzione ed una trasparente governance, opera citata, punto 4.1 .

${ }^{56}$ Tali modifiche sono previste dal Piano triennale d'azione 2011 - 2013 della Strategia intersettoriale per la prevenzione e la lotta alla corruzione ed una trasparente governance, e devono essere realizzate entro il 2012. Per maggiori informazioni v. il Piano triennale d'azione 2011 - 2013, opera citata, punto 3.

${ }^{57}$ Relazione di Valutazione per l'Albania, approvato da GRECO nel corso della seconda seduta plenaria di Strasburgo, 9 - 13 Dicembre 2003.

${ }^{58}$ Dal sondaggio dell'IDRA per il 2009, l'unica istituzione apprezzata dal pubblico come strumento d'aiuto al contrasto della corruzione, aggiudicata con 63.6 punti, sono i media. Tale percezione e' migliorata con 4.2 punti rispetto al sondaggio del 2005. Per maggiori informazioni v. IDRA surve, 2009, opera citata, pg. 13. 
consapevolezza cittadina contro di esso. L'attivita' della societa' civile si è principalmente concentrata sul monitoraggio dell'applicazione dei provvedimenti anticorruzione e sulla realizzazione di sondaggi sulla manifestazione della corruzione. Nonostante tutto cio', la societa' civile deve essere piu' attiva al fine di aumentare la pressione sulle strutture statali e la credibilita' del pubblico.

La lotta alla corruzione è una lotta multidimensionale che si protrae nel tempo. Ovvio che le riforme sopra elencate non possono eliminare la corruzione pero' possono incidere a minimizzarla e tenerla sotto controllo perche' il Paese vada avanti negli sforzi ad essere un paese europeo, sviluppato e democratico.

Essendo un fenomeno che direttamente 0 indirettamente interessa tutta la societa', la lotta al fenomeno richiede la partecipazione di tutti i suoi componenti ed il coordinamento dell'attivita' di tutti gli attori all'attuazione delle politiche contro tale fenomeno.

\section{References}

Il Codice Penale della Repubblica di Albania, approvato con la Legge nr. 1470 del 12.05.1952.

Il Codice Penale della Repubblica di Albania, approvato con la Legge nr. 5591 del 15.06.1977.

II Codice Penale della Repubblica di Albania, approvato con la Legge 7895 del 27.01.1995

Il Codice di Procedura Penale, Centro delle pubblicazioni ufficiali, 2002

Ismet E. Lo sviluppo storico della legislazione penale in Albania, 1998, albin, Tirana

Ismet E. Diritto Penale, Parte Speciale, 2002, SHBLU, Tirana

Ismet E. Commentario degli atti aggiuntivi e delle modifiche apportate al Codice Penale, 2004, albin, Tirana

Hysi V., (2007), "La politica penale albanese nell'ambito dei reati di corruzione", studi giuridici, nr. 1, Pegi, Tirana

"L'aumento dell'accesso dei cittadini agli organi di giustizia per una efficacia applicazione della legislazione albanese quale importante strumento alla lotta contro la corruzione", Pubblicazione del Centro per l'iniziativa legislativa e cittadina, Tirana, giugno 2007.

U, Mynit, Corruption, Causes, consequences and cures, , Asia Pacific Development Juornal, Vol 7, No.2, December 2000

Daniela Irrera, The balkanisation of politics, crime and coruption in Albania, Europian University Institutee, EUI Working Paper, RSCAS 2006

Swedish Internatioanl Development Cooperation Agency (SIDA), Albanian Anticoruption study, Ramboll Manegement, 2008

Relazione di Valutazione per l'Albania, approvata da GRECO nel corso della seconda riunione plenaria a Strasburgo, 9 - 13 dicembre 2003.

Transparency International Albania, EU anticorruption requirements, measuring progress in Albania, 2011

Transparency International, Interantional Secretariat, Corruption Perception Index 2011,

Europian Commision, Albania 2011 Progress Report, Commission staff working paper, Brussels, 12.10.2011, sec(2011) 1205

La corruzione in Albania, percezioni ed esperienze, Institute for Development Research and alternatives, (IDRA) 2009

La Legge nr. 8635 del 06/07/2000 Sulla ratifica della Convenzione civile "Sulla corruzione"

La Legge nr. 878 del 26.04.2001 Sulla ratifica della Convenzione penale "Sulla corruzione"

La Legge nr. 9492 del 13.03.2006 Sulla ratifica della Convenzione delle Nazioni Unite "Contro la corruzione"

La Legge nr. 9245 del 24.06.2004 Sulla ratifica del "Protocollo aggiuntivo della Convenzione penale sulla corruzione"

La Legge "Sulle gare pubbliche" nr. 9643 del 20.11.2006, modificata

Le Legge nr. 9508 del 03.04.2006 „Sulla collaborazione del pubblico alla lotta contro la corruzione“

La Legge nr. 9367 del 07.04.2005 "Sulla prevenzione del conflitto d'interesse nell'esercizio delle funzioni pubbliche", modificata

La Legge nr. 9049 del 10.04.2003 "Sulla dichiarazione ed il controllo dei beni, degli obblighi finanziari degli eletti e di alcuni pubblici ufficiali", art. 1

Consiglio dei Ministri, il Piano Nazionale per l'attuazione dell'Accordo di Stabilizzazione Associazione, la lotta alla corruzione, Giugno, Tirana 2006

Delibera nr. 1561 del 03.10.2008 "Sull'approvazione della strategia intersettoriale per la prevenzione e la lotta della corruzione e per una trasparente governance, 2008 - 2013"

II Piano triennale 2011 - 2013 della Strategia Intersettoriale per la Prevenzione, la Lotta alla Corruzione e per una Trasparente Governance, approvato nel corso della riunione del Gruppo di Lavoro Interministeriale in data 8 giugno 2011, punto 6. 
